presentations with handouts, ahead of the January 2021 examinations. Content was based on national guidelines and published research. 5 sessions were delivered with the final session including guest consultant panellists to answer trainee questions. Quantitative and qualitative feedback was collected from the attendees.

Result. 172 doctors registered onto the course, with 44 NHS trusts represented. Doctors from a variety of grades attended, with 55\% CT3s, $17 \%$ Specialty Doctors, $16 \%$ CT2s, $8 \%$ CT1s, $4 \%$ in other roles. $100 \%$ of attendees stated that they would recommend the course to any doctor sitting the CASC. $97 \%$ of attendees rated the course as either 'Excellent' or 'Good'. Qualitative feedback was positive and 3 themes were identified- communication, content and the online format.

Conclusion. The CASC course provided an opportunity to deliver national teaching to trainees based on national guidelines and peer-reviewed research, with a focus on addressing areas that trainees may feel less confident with. The course received significant positive feedback from attendees. The significant number of pre-CT3 trainees attending the course suggests that there may be an interest from this group for further support in developing the complex communication skills that ultimately are assessed by the CASC exam.

An innovative CASC training redesign - 'experience of virtual mock CASC exam'

Jennifer Rankin ${ }^{1 \star}$, Jessica Foster ${ }^{2}$ and Omer Minhas ${ }^{3}$

${ }^{1}$ Aneurin Bevan UHB; ${ }^{2}$ Cardiff \& Vale UHB and ${ }^{3}$ Tonteg Hospital ${ }^{*}$ Corresponding author.

doi: $10.1192 /$ bjo.2021.428

Aims. Cardiff CASC Training (CCT) has provided structured and formal training for the CASC exam for Wales trainees since 2012, in conjunction with Wales Deanery. For the past 8 years CCT has delivered face-to-face mock CASC exams and received excellent feedback from candidates and examiners, in addition to an extremely positive outcome of improvement in CASC pass rate for candidates.

Due to the current COVID-19 pandemic restrictions delivery of the mock CASC examination had to be adapted with the aim of running it remotely via an online platform.

Method. The examinations were run online via Zoom due to its ease of use, including the ability to screen share candidate instructions and assign participants in to breakout rooms. One lead exam coordinator manually rotated candidates around the circuit of 16 stations.

Result. A total of 16 candidates sat the mock exam over two separate sittings. Written feedback was obtained from candidates and examiners. Limitations identified during the initial sitting included high logistical workload for the lead exam co-ordinator and Zoom not being supported by all hospital computer internet browsers, these issues were addressed prior to the second sitting. Feedback from candidates regarding the overall experience of the online exams ranged from 'extremely effective' to 'very effective', this is in line with feedback obtained following previous face-to-face mock exam events CCT has run.

Conclusion. Although online learning may feel very different to the face-to-face interactions we are all used to we are in an era where adaption is necessary. These online mock CASC examinations have been a success and are also in keeping with how the real CASC examination is currently being run by the Royal College of Psychiatrists. CCT are running a further online mock examination to support the next cohort of candidates through their CASC exam in this particularly challenging time.

\section{Peer-led medication education in the time of COVID-19}

Rosa Roberts*

Avon and Wiltshire Partnership Trust; Barnet Enfield and Haringey Mental Health NHS Trust

${ }^{\star}$ Corresponding author.

doi: 10.1192/bjo.2021.429

Aims. To set up an online peer-led medical education programme for core psychiatry trainees during the COVID-19 pandemic.

To determine trainees' views regarding the role of peer-led education in psychiatry.

Method. A peer-led education programme was set up for psychiatry trainees in their third year of core training, held over an online video-conferencing platform. The weekly sessions were organised and led by trainees. Each week a trainee either presented a journal article or a particular psychiatric topic, with a group discussion afterwards.

An online survey was sent to psychiatry trainees that had participated in the programme to determine their views. Close-ended questions were asked as well as open-ended questions for more qualitative responses.

Result. There were 9 peer-led sessions in total, with 11 trainees (out of 18 invited) attending at least one session, and an average of 5 trainees at each session.

Five core trainee psychiatrists responded to the survey following the sessions. All of the respondents found the sessions either "very" or "fairly" useful. $80 \%$ stated that there should be more peer teaching opportunities as part of normal psychiatry training. All respondents found engaging with online teaching either "easy" or "OK".

Open-ended questions showed that respondents found the sessions were useful for: 1) connecting with peers during a difficult time 2) free discussion due to being around peers 3) wide interest and variety of topics. Improvements that could have been made were: 1) more sessions 2) advance knowledge of journal articles being presented.

Conclusion. Peer-led sessions are a useful form of medical education for core psychiatry trainees. During the restrictions brought about by the COVID-19 pandemic they are a way to connect with colleagues during an isolating time. They are easy to organise and access; and can take pressure off medical institutions, whilst having the advantages of allowing trainees to feel they can discuss topics in a more open manner and can follow their own interests.

\section{Immersive psychiatry simulation: a novel course for medical student training}

Kenneth Ruddock* ${ }^{\star}$ Kim Herbert, Catriona Neil, Neera Gajree and Karli Dempsey

NHS Lanarkshire

${ }^{\star}$ Corresponding author.

doi: 10.1192/bjo.2021.430

Aims. Over the last decade, there have been significant developments in the use of simulation for undergraduate medical education. Despite simulation's diverse applications across the medical school curriculum, it has thus far been underutilised within psychiatry teaching. Psychiatric simulation can support students to develop strategies to elicit psychopathology, de-escalate an aggressive patient or perform a risk assessment. Such experiences can be difficult to encounter during clinical placements and may 